\title{
Ischemic preconditioning protects neurons from damage and maintains the immunoreactivity of kynurenic acid in the gerbil hippocampal CA1 region following transient cerebral ischemia
}

\author{
JAE-CHUL LEE $^{1 *}$, HYUN-JIN TAE ${ }^{2 *}$, GEUM-SIL CHO ${ }^{3}$, IN HYE KIM ${ }^{1}$, JI HYEON AHN ${ }^{1}$, \\ JOON HA PARK ${ }^{1}$, BAI HUI CHEN ${ }^{4}$, JEONG-HWI CHO ${ }^{1}$, BICH NA SHIN ${ }^{4}$, JUN HWI CHO ${ }^{5}$, EUN JOO BAE ${ }^{6}$, \\ JINSEU PARK ${ }^{2}$, YOUNG-MYEONG $\mathrm{KIM}^{7}$, SOO YOUNG CHOI ${ }^{2}$ and MOO-HO WON ${ }^{1}$ \\ ${ }^{1}$ Department of Neurobiology, School of Medicine, Kangwon National University, Chuncheon 200-701; \\ ${ }^{2}$ Department of Biomedical Science and Research Institute for Bioscience and Biotechnology, Hallym University, \\ Chuncheon 200-702; ${ }^{3}$ Department of Neuroscience, College of Medicine, Korea University, Seoul 136-705; \\ ${ }^{4}$ Department of Physiology, College of Medicine, and Institute of Neurodegeneration and Neuroregeneration, \\ Hallym University, Chuncheon 200-702; ${ }^{5}$ Department of Emergency Medicine, School of Medicine, \\ Kangwon National University; ${ }^{6}$ Department of Pediatrics, Chuncheon Sacred Heart Hospital, \\ College of Medicine, Hallym University; ${ }^{7}$ Department of Molecular and Cellular Biochemistry, \\ School of Medicine, Kangwon National University, Chuncheon 200-701, Republic of Korea
}

Received October 2, 2014; Accepted April 3, 2015

DOI: $10.3892 / \mathrm{ijmm} .2015 .2171$

\begin{abstract}
Pyramidal neurons in region I of hippocampus proper (CA1) are particularly vulnerable to excitotoxic processes following transient forebrain ischemia. Kynurenic acid (KYNA) is a small molecule derived from tryptophan when this amino acid is metabolized through the kynurenine pathway. In the present study, we examined the effects of ischemic preconditioning (IPC) on the immunoreactivity and protein levels of KYNA following 5 min of transient forebrain ischemia in gerbils. The animals were randomly assigned to 4 groups (sham-operated group, ischemia-operated group, IPC + sham-operated group and IPC + ischemia-operated group). IPC was induced by subjecting the gerbils to $2 \mathrm{~min}$ of ischemia followed by 1 day of recovery. In the ischemia-operated group, we observed a significant loss of pyramidal neurons in the CA1
\end{abstract}

Correspondence to: Professor Moo-Ho Won, Department of Neurobiology, School of Medicine, Kangwon National University, 1 Kangwondaehak-gil Chuncheon 200-701, Republic of Korea

E-mail: mhwon@kangwon.ac.kr

Professor Soo Young Choi, Department of Biomedical Science and Research Institute of Bioscience and Biotechnology, Hallym University, 1 Hallymdaehak-gil, Chuncheon 200-702, Republic of Korea

E-mail: sychoi@hallym.ac.kr

*Contributed equally

Key words: ischemic preconditioning, transient ischemia, CA1 pyramidal neurons, delayed neuronal death, kynurenic acid stratum pyramidale (SP) at 5 days post-ischemia; however, in the IPC + ischemia-operated group, the pyramidal neurons were well protected. KYNA immunoreactivity in the SP of the ischemia-operated group was significantly altered following ischemia-reperfusion and was very low 5 days following ischemia-reperfusion. In the IPC + ischemia-operated group, however, KYNA immunoreactivity was constitutively detected in the SP of the CA1 region after the ischemic insult. We also found that the alteration pattern of the KYNA protein level in the CA1 region following ischemia was generally similar to the immunohistochemical changes observed. In brief, our findings demonstrated that IPC maintained and even increased KYNA immunoreactivity in the SP of the CA1 region following ischemia-reperfusion. The data from the present study thus indicate that the enhancement of KYNA expression by IPC may be necessary for neuronal survival following transient ischemic injury.

\section{Introduction}

Transient forebrain ischemia induced by the deprivation of brain blood flow leads to irreversible brain damage in specific vulnerable areas of the brain (1-3). Delayed neuronal death occurs several days after ischemia-reperfusion injury in the stratum pyramidale (SP) of region I of hippocampus proper (CA1) (4-7). One of the mechanisms of delayed neuronal death is possibly associated with a number of biochemical events triggered by glutamate excitotoxicity (8-10).

Ischemic preconditioning (IPC) represents an important adaptation of the central nervous system (CNS) to sublethal ischemia, which can increase the ischemic tolerance of the CNS to a subsequent longer or lethal period of ischemia $(11,12)$. IPC induces the expression of diverse genes involved in 
cytoprotection and, in turn, encodes proteins that lead to the enhancement of resistance to cerebral ischemia (13). This phenomenon is termed cerebral 'ischemic tolerance', although the basic mechanisms underlying cerebral ischemic tolerance are not yet fully understood (14).

Kynurenic acid (KYNA) is an endogenous metabolite of the kynurenine pathway for tryptophan degradation and is produced from its precursor L-kynurenine (KYN) by the enzyme, kynurenine-aminotransferase (15). The formation of KYNA in particular, has been shown to play an important role in the CNS at physiological concentrations, as this metabolite selectively acts as an antagonist of N-methyl-D-aspartate (NMDA) receptors by blocking the co-agonist site for glycine $(16,17)$, as well as a non-competitive inhibitor of $\alpha 7$-nicotinic receptors for acetylcholine (18). Thus, KYNA protects neuronal cells from excitotoxicity evoked by the overactivation of NMDA receptors. Furthermore, KYNA plays a versatile role in pathological states, including inflammatory (19), vascular (20) and antioxidant (21) processes. Indeed, the exogenous administration of KYNA or its enhanced endogenous synthesis has been proven to be a robust neuroprotectant in animal models of forebrain ischemia (22-25). However, research on KYNA as a neuroprotective agent is rather limited, as it hardly crosses the blood-brain barrier $(22,26)$.

To the best of our knowledge, the expression patterns of endogenous KYNA immunoreactivity in the IPC-mediated hippocampus following transient forebrain ischemia have not been studied thus far. Therefore, the present study was carried out to investigate the temporal changes and specific roles of endogenous KYNA in the IPC-induced neuroprotective effects against transient ischemic damage in the hippocampus of gerbils, which are considered to be a good animal model for studying transient cerebral ischemia $(27,28)$.

\section{Materials and methods}

Experimental animals. We used the progeny of male Mongolian gerbils (Meriones unguiculatus) obtained from the Experimental Animal Center, Kangwon National University, Chuncheon, Korea. The gerbils were used at 24 weeks of age (body weight, 65-75 g) and were maintained under pathogenfree conditions with a temperature of $23^{\circ} \mathrm{C}$ and a humidity of $60 \%$. All the experimental protocols were approved by the Institutional Animal Care and Use Committee (IACUC) at Kangwon National University and adhered to guidelines that are in compliance with the current international laws and policies (Guide for the Care and Use of Laboratory Animals, The National Academies Press, 8th edition, 2011).

Animal groups and induction of transient forebrain ischemia. The animals were divided into 4 groups $(n=7$ at each time point): i) group 1, the sham-operated group: the bilateral common carotid arteries were exposed and the animals were not subjected to ischemia (sham-operation); ii) group 2 , the ischemia-operated group: the animals were subjected to $5 \mathrm{~min}$ of transient forebrain ischemia; iii) group 3 , the IPC + sham-operated group: the animals were subjected to 2 min of sublethal ischemia prior to sham-operation; and iv) group 4, the IPC + ischemia-operated group: the animals were subjected to $2 \mathrm{~min}$ of sublethal ischemia prior to $5 \mathrm{~min}$ of transient ischemia. The IPC paradigm has been proven to be very effective at protecting neurons against ischemic damage in this ischemic model (29). The animals in groups 2 and 4 were allowed to recover for different periods of time (sham, 1 day, 2 days and 5 days), as pyramidal neurons in the hippocampal CA1 region survive for 3 days and then begin to die 4-5 days following ischemia-reperfusion.

Transient forebrain ischemia was developed according to the method described in our previous study (30). In brief, the experimental animals were anesthetized with a mixture of $2.5 \%$ isoflurane in $33 \%$ oxygen and $67 \%$ nitrous oxide. Ischemia was induced by the occlusion of arteries with non-traumatic aneurysm clips (Yasargil FE 723K; Aesculap, Tuttlingen, Germany). After 2 or 5 min of occlusion, the aneurysm clips were removed from the common carotid arteries. The body (rectal) temperature under free-regulating or normothermic $\left(37 \pm 0.5^{\circ} \mathrm{C}\right)$ conditions was monitored with a rectal temperature probe (TR-100; Fine Science Tools, Foster City, CA, USA) and maintained using a thermometric blanket before, during and after surgery until the animals had completely recovered from the anesthesia. Thereafter, the animals were kept in a thermal incubator (temperature, $23^{\circ} \mathrm{C}$; humidity, 60\%) (Mirae Medical Industry, Seoul, Korea) to maintain the body temperature of the animals until they were sacrificed (as described below).

Tissue processing for histological analysis. For histological analysis, the gerbils ( $n=7$ at each time point) were deeply anesthetized with pentobarbital sodium and perfused through the left ventricle with $0.1 \mathrm{M}$ phosphate-buffered saline (PBS, $\mathrm{pH}$ 7.4) followed by $4 \%$ paraformaldehyde in $0.1 \mathrm{M}$ phosphatebuffer (PB, $\mathrm{pH}$ 7.4). The brains were removed and post-fixed in the same fixative for $6 \mathrm{~h}$. The brain tissues were embedded in tissue-freezing medium and serially sectioned into $30-\mu \mathrm{m}$ coronal sections using a cryostat (Leica, Wetzlar, Germany).

Cresyl violet $(C V)$ staining and Fluoro-Jade B (F-J B) histofluorescence. To investigate the delayed neuronal damage in the hippocampus following ischemia-reperfusion, CV staining and F-J B histofluorescence were performed as previously described (31). In brief, the sections were stained with $1.0 \%(w / v)$ CV acetate (Sigma-Aldrich, St. Louis, MO, USA) and dehydrated. They were then mounted with Canada balsam (Kanto Chemical Co., Tokyo, Japan). For F-J B histofluorescence, the sections were immersed in a $0.0004 \%$ F-J B staining solution (Histochem Inc., Jefferson, AR, USA). After washing, the sections were examined using an epifluorescent microscope (Carl Zeiss, Göttingen, Germany) with blue (450-490 nm) excitation light and a barrier filter.

Immunohistochemistry for neuronal nuclei (NeuN) and KYNA. For immunohistochemical staining, the sections were prepared out according to the method described in our previous study (31). The brain sections were blocked with $10 \%$ normal goat serum in $0.05 \mathrm{M}$ PBS followed by staining with primary mouse antiNeuN antibody (a neuron-specific soluble nuclear antigen) (diluted 1:1,000; MAB377; Chemicon International, Temecula, CA, USA) and rabbit anti-KYNA antibody (diluted 1:200; ab37105; Abcam, Cambridge, MA, USA) overnight at $4^{\circ} \mathrm{C}$. The sections were then incubated with the secondary antibodies (BA-9200, BA-1000; Vector Laboratories Inc., Burlingame, CA, 
USA) and were developed using the Vectastain ABC system (Vector Laboratories Inc.). Subsequently, they were visualized with 3,3'-diaminobenzidine in $0.1 \mathrm{M}$ Tris- $\mathrm{HCl}$ buffer. In order to establish the specificity of the immunostaining, a negative control test was carried out with pre-immune serum instead of primary antibody. The negative control resulted in the absence of immunoreactivity in any structures.

Western blot analysis. To obtain the accurate data for changes in the protein levels of KYNA in the hippocampal CA1 region following transient forebrain ischemia abd IPC, the animals ( $n=7$ at each time point) were sacrificed at designated time points (sham, 1, 2 and 5 days) following ischemia-reperfusion and the brain tissues were used for western blot analysis. As previously described (31), after the animals were sacrificed, the brain tissues were removed and transversely cut into sections with a thickness of $400 \mu \mathrm{m}$ using a vibratome (Leica), and the hippocampal CA1 region was dissected with a surgical blade, removing the hippocampus. The tissues were homogenized in $50 \mathrm{mM}$ PBS (pH 7.4) containing $0.1 \mathrm{mM}$ ethylene glycolbis(2-aminoethyl Ether)-N,N,N',N' tetraacetic acid (EGTA; $\mathrm{pH}$ 8.0), 0.2\% Nonidet P-40, $10 \mathrm{mM}$ ethylendiaminetetraacetic acid (EDTA; pH 8.0), $15 \mathrm{mM}$ sodium pyrophosphate, $100 \mathrm{mM}$ $\beta$-glycerophosphate, $50 \mathrm{mM} \mathrm{NaF}, 150 \mathrm{mM} \mathrm{NaCl}, 2 \mathrm{mM}$ sodium orthovanadate, $1 \mathrm{mM}$ phenylmethylsulfonyl fluoride (PMSF) and $1 \mathrm{mM}$ dithiothreitol (DTT). Following centrifugation (at 12,000 rpm for $10 \mathrm{~min}$ ), the protein level was determined in the supernatants using a Micro BCA protein assay kit with bovine serum albumin as the standard (Pierce Chemical Co., Rockford, IL, USA). Aliquots containing $20 \mu \mathrm{g}$ of total protein were boiled in loading buffer containing $150 \mathrm{mM}$ Tris (pH 6.8), 3 mM DTT, 6\% SDS, 0.3\% bromophenol blue and $30 \%$ glycerol. Subsequently, each aliquot was loaded onto a $12.5 \%$ polyacryamide gel. Following electrophoresis, the gels were transferred onto nitrocellulose transfer membranes (Pall Corp., East Hills, NY, USA). To reduce background staining, the membranes were incubated with $5 \%$ non-fat dry milk in PBS containing 0.1\% Tween-20 for 45 min, and then with rabbit anti-KYNA antibody (diluted 1:1,500; Santa Cruz Biotechnology) and peroxidase-conjugated goat anti-rabbit IgG (A0545; Sigma-Aldrich), and then subjected to enhanced chemiluminescence using an ECL kit (Pierce Chemical Co.). Loading controls were performed using antibodies against $\beta$-actin (Abcam, Inc., Cambridge, MA, USA).

Data analysis. The brain tisue sections were selected according to anatomical landmarks corresponding to target coordinates [anteroposterior (AP) diameter from -1.4 to $-1.8 \mathrm{~mm}$ ] of the gerbil brain atlas. The number of $\mathrm{CV}$-positive, $\mathrm{NeuN}$-immunoreactive and F-J B-positive cells was counted in a 200x200 $\mu \mathrm{m}^{2}$ area, applied approximately at the center of the CA1 in the SP. Cell counts were obtained by averaging the total cell numbers from each animal per group.

In order to quantitatively analyze KYNA immunoreactivity, we applied a method described in a previous study of ours (27). In brief, the density of immunoreactive structures was evaluated on the basis of a relative optical density (ROD), which was obtained after the transformation of the mean gray level using the following formula: ROD $=\log (256 /$ mean gray level).
According to a method described in a previous study of ours (28), the results of western blot analysis were quantified using Scion Image software (Scion Corp., Frederick, MD, USA), which was used to count the ROD: a ratio of the ROD was calibrated as a percentage, with the value in the shamoperated group designated as $100 \%$.

Statistical analysis. All data are presented as the means \pm SEM. A multiple-sample comparison was applied to examine the differences between groups and days. The differences between groups on the same day were assessed by one-way ANOVA and Tukey's post-hoc test. For the analysis of time-dependent differences between the groups, two-way ANOVA with the Bonferroni post-hoc test were used. A p-value $\leq 0.05$ was considered to indicate a statistically significant difference.

\section{Results}

\section{IPC-mediated neuroprotection}

$C V$-positive $\left(\mathrm{CV}^{+}\right)$cells. We examined whether IPC is associated with a decrease in neuronal damage/death in the gerbil hippocampus following ischemia-reperfusion. $\mathrm{CV}^{+}$cells were clearly observed in all the subregions of the hippocampus in the sham-operated group, and the neurons in the SP (pyramidal neurons) had a slightly large, round or pyramid-like shaped morphology (Fig. 1A and B). In the ischemia-operated group, however, 5 days after ischemia-reperfusion, the $\mathrm{CV}^{+}$cells were significantly damaged in the SP of the CA1 region, but not in the $\mathrm{CA} 2 / 3$ region, compared with those of the sham-operated group (Fig. 1E); the damaged cells were shrunken and contained dark and polygonal nuclei (arrows in Fig. 1F).

In the IPC + sham-operated group, the CA1 pyramidal neurons were evidently stained with CV (Fig. 1C and D), and in the IPC + ischemia-operated group, the distribution pattern of the $\mathrm{CV}^{+}$cells in the SP was very similar to that in the IPC + sham-operated group at 5 days following ischemia-reperfusion (Fig. $1 \mathrm{G}$ and $\mathrm{H}$ ).

$\mathrm{NeuN}^{+}$and $\mathrm{F}-\mathrm{JB} \mathrm{B}^{+}$cells. The assessment of the IPC-mediated neuroprotective effects in the CA1 region was carried out using anti-NeuN immunohistochemistry and F-J B histofluorescence staining (Fig. 2). In the sham-operated group, the pyramidal neurons in the CA1 region were evidently stained with $\mathrm{NeuN}$, and no F-J B ${ }^{+}$neurons were observed (Table I and Fig. 2A and $\mathrm{B})$. In the ischemia-operated group, however, the number of $\mathrm{NeuN}^{+}$neurons was significantly decreased in the SP of the CA1 region 5 days following ischemia-reperfusion (Table I and Fig. 2E), and, at this time point, many F-J B $\mathrm{B}^{+}$cells were observed in the SP of the CA1 region (Table I and Fig. 2F).

In the IPC + sham-operated group, the pyramidal neurons in the CA1 region were also evidently stained with NeuN (Fig. 2C), and no F-J B ${ }^{+}$cells were observed (Table I and Fig. 2D). In the IPC + ischemia-operated group, the distribution patterns of the $\mathrm{NeuN}^{+}$and F-J B ${ }^{+}$cells in the SP were not significantly altered compared with those in the IPC + sham-operated group (Table I and Fig. 2G and H).

\section{IPC-mediated effect on KYNA immunoreactivity}

$C A 1$ region. KYNA immunoreactivity was easily detected in the SP of the CA1 region in the sham-operated group (Table II and Fig. 3A, panels a-h). In the ischemia-operated group, we 


\section{Ischemia \\ Ischemia}
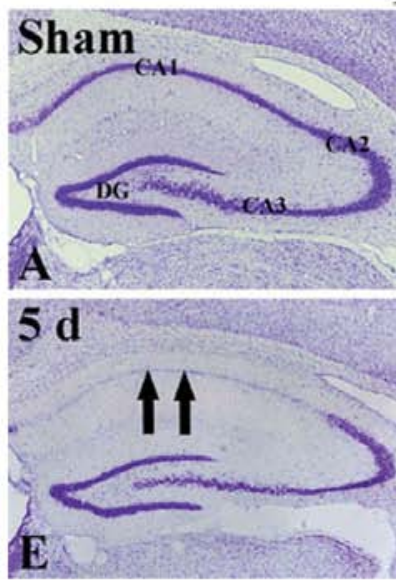
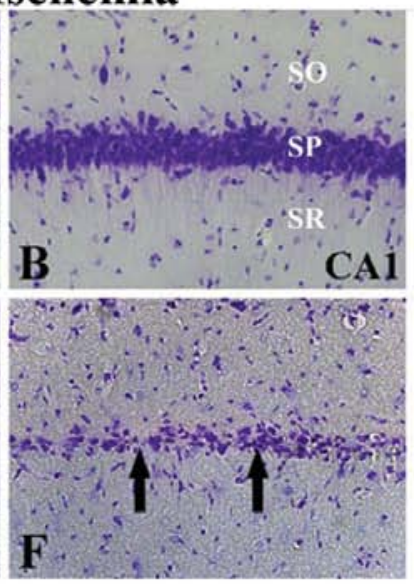

IPC+Ischemia

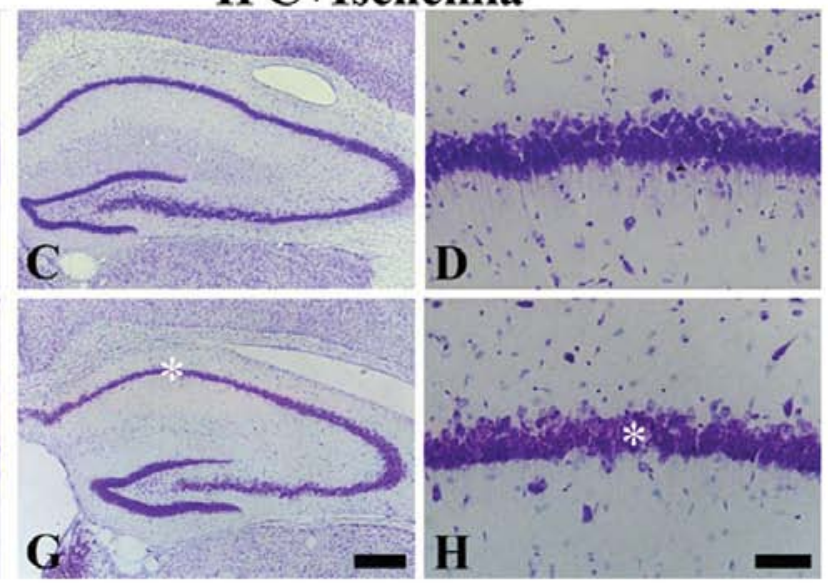

Figure 1. CV staining in the hippocampus of the ischemia-operated (left 2, columns) and IPC + ischemia-operated (right 2 columns) groups at (A-D) sham and (E-H) 5 days $(5 \mathrm{~d})$ post-ischemia. $\mathrm{CV}^{+}$cells (arrows) are damaged in the stratum pyramidale (SP) of the CA1 region only at 5 days post-ischemia in the ischemiaoperated group; however, $\mathrm{CV}^{+}$cells (asterisk) in the IPC + ischemia-operated group were similar to those in the sham-operated group. SO, stratum oriens; SR, stratum radiatum; IPC, ischemic preconditioning; DG, dentate gyrus; CA1, CA2 and CA3 represent regions I, II and III of hippocampus proper, respectively; CV, cresyl violet. Scale bar: (A, C, E and G) $800 \mu \mathrm{m}$ and (B, D, F and H) $50 \mu \mathrm{m}$.
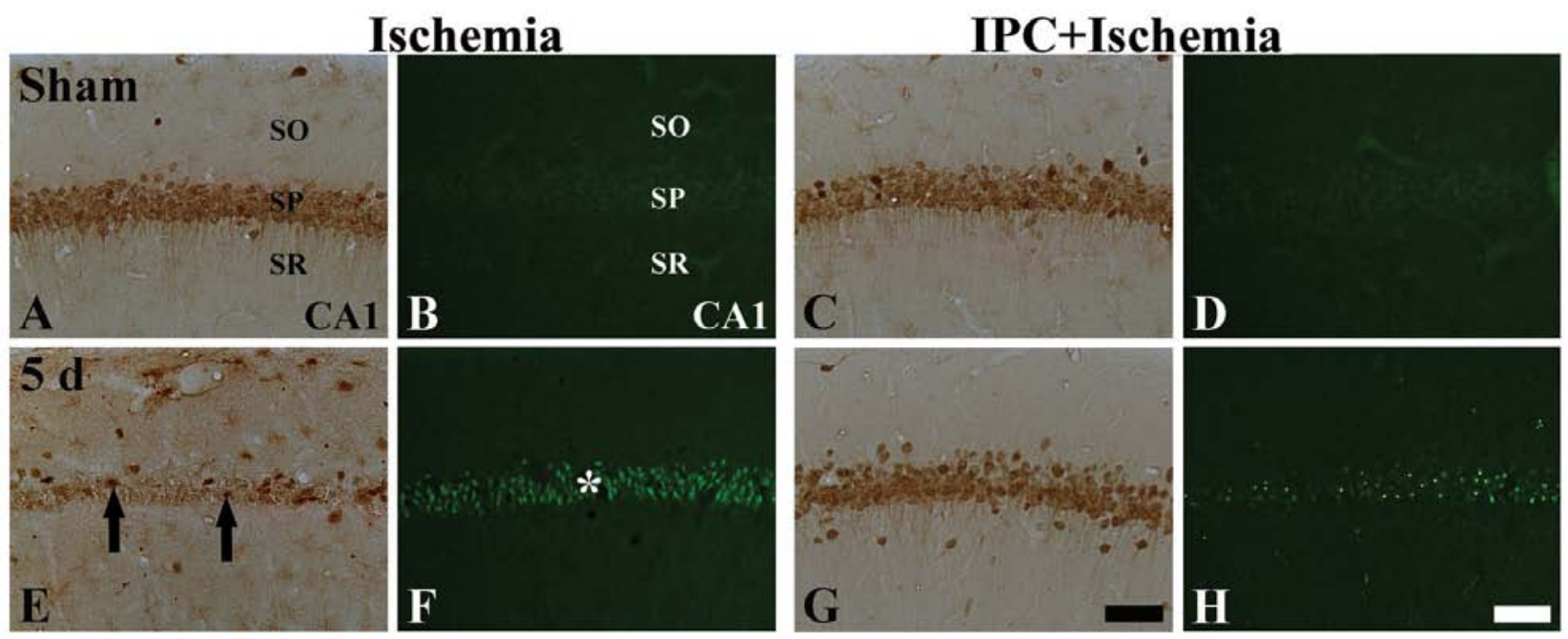

Figure 2. NeuN immunohistochemistry (the first and third longitudinal columns) and F-J B histofluorescence staining (the second and fourth longitudinal columns) in the CA1 region of the ischemia-operated (left 2 columns) and IPC + ischemia-operated (right 2 columns) groups at (A-D) sham and (E-F) 5 days ( 5 d) following ischemia-reperfusion. In the sham-operated group, many $\mathrm{NeuN}^{+}$neurons, but no F-J $\mathrm{B}^{+}$cells were observed in the stratum pyramidale (SP). In the ischemia-operated group, a few NeuN ${ }^{+}$(black arrows) and many F-J B ${ }^{+}$(asterisk) cells were detected in the SP at 5 days post-ischemia. However, in the IPC + ischemia-operated group, abundant $\mathrm{NeuN}^{+}$and few F-J B ${ }^{+}$cells were detected in the SP at 5 days post-ischemia. NeuN, neuronal nuclei; F-J B, Fluoro-Jade B; CA1, region I of hippocampus proper; IPC, ischemic preconditioning; SO, stratum oriens; SR, stratum radiatum. Scale bar, $50 \mu \mathrm{m}$.

found that KYNA immunoreactivity was altered in the SP following ischemia-reperfusion. The immunoreactivity was significantly increased 1 day following ischemia-reperfusion and decreased at 2 days post-ischemia (Table II and Fig. 3A, panels $\mathrm{c}$ and e). Five days after ischemia, KYNA immunoreactivity was even more decreased in the SP (Table II and Fig. 3A, panel $g$ ).

In the IPC + sham-operated group, KYNA immunoreactivity was similar to that in the sham-operated group (Table II and Fig. 3A, panel b). In the IPC + ischemia-operated group, KYNA immunoreactivity in the SP was a slightly increased at 1 day post-ischemia, and, thereafter, KYNA immunoreactivity in the SP was significantly higher than that in the IPC + shamoperated group (Table II and Fig. 3A, panels d, f and h).

$C A 2 / 3$ region. In the $\mathrm{CA} 2 / 3$ region of the hippocampus of the brains of the gerbils in the sham-operated group, moderate KYNA immunoreactivity was detected in neurons of the SP (Table II and Fig. 3B, panel a). KYNA immunoreactivity was not significantly altered in the SP following ischemiareperfusion (Table II and Fig. 3B panels c, e and g).

In the IPC + sham-operated and ischemia-operated groups, KYNA immunoreactivity in the SP in the CA1 region was similar to that in the sham-operated group (Table II and Fig. 3B panels $b, d$, f and $h$ ). 
Table I. Changes in the mean number of pyramidal neurons of the hippocampal CA1 region in the ischemia-operated and IPC + ischemia-operated gerbils.

\begin{tabular}{|c|c|c|c|c|}
\hline \multirow[b]{2}{*}{ Time after IR } & \multicolumn{2}{|c|}{ Ischemia-operated group } & \multicolumn{2}{|c|}{ IPC + ischemia-operated group } \\
\hline & $\mathrm{NeuN}^{+}$ & F-J B ${ }^{+}$ & $\mathrm{NeuN}^{+}$ & F-J B ${ }^{+}$ \\
\hline Sham & $354 \pm 14.14$ & 0 & $344 \pm 12.36$ & 0 \\
\hline 1 day & $364 \pm 16.88$ & 0 & $351 \pm 15.66$ & 0 \\
\hline 2 days & $359 \pm 13.65$ & $5 \pm 3.72 \mathrm{a}$ & $357 \pm 16.71$ & 0 \\
\hline 5 days & $38 \pm 14.36^{\mathrm{a}, \mathrm{b}}$ & $141 \pm 15.77^{\mathrm{a}, \mathrm{b}}$ & $331 \pm 15.65^{\mathrm{a}, \mathrm{b}}$ & $17 \pm 6.74^{\mathrm{a}, \mathrm{b}}$ \\
\hline
\end{tabular}
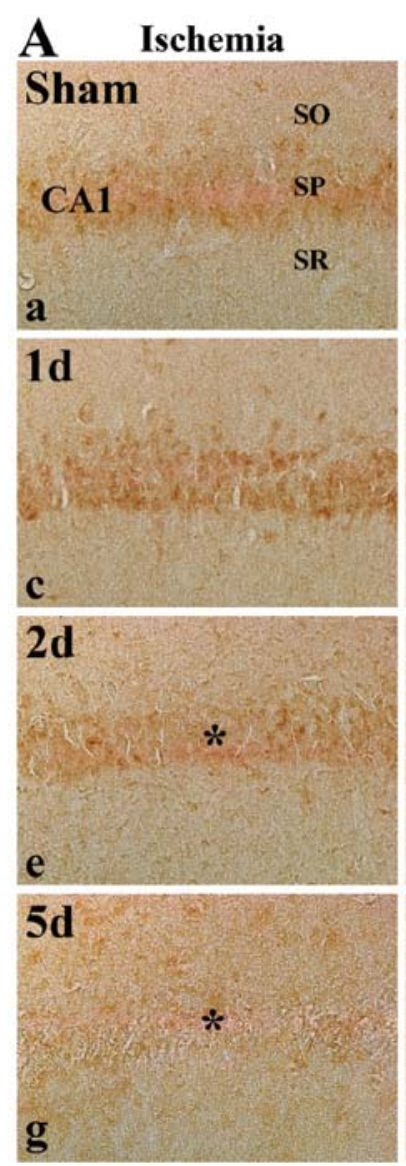
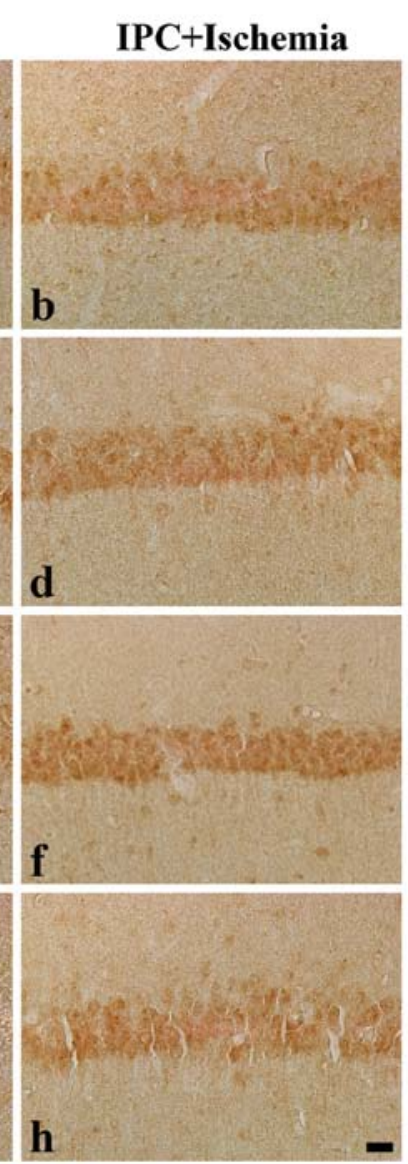
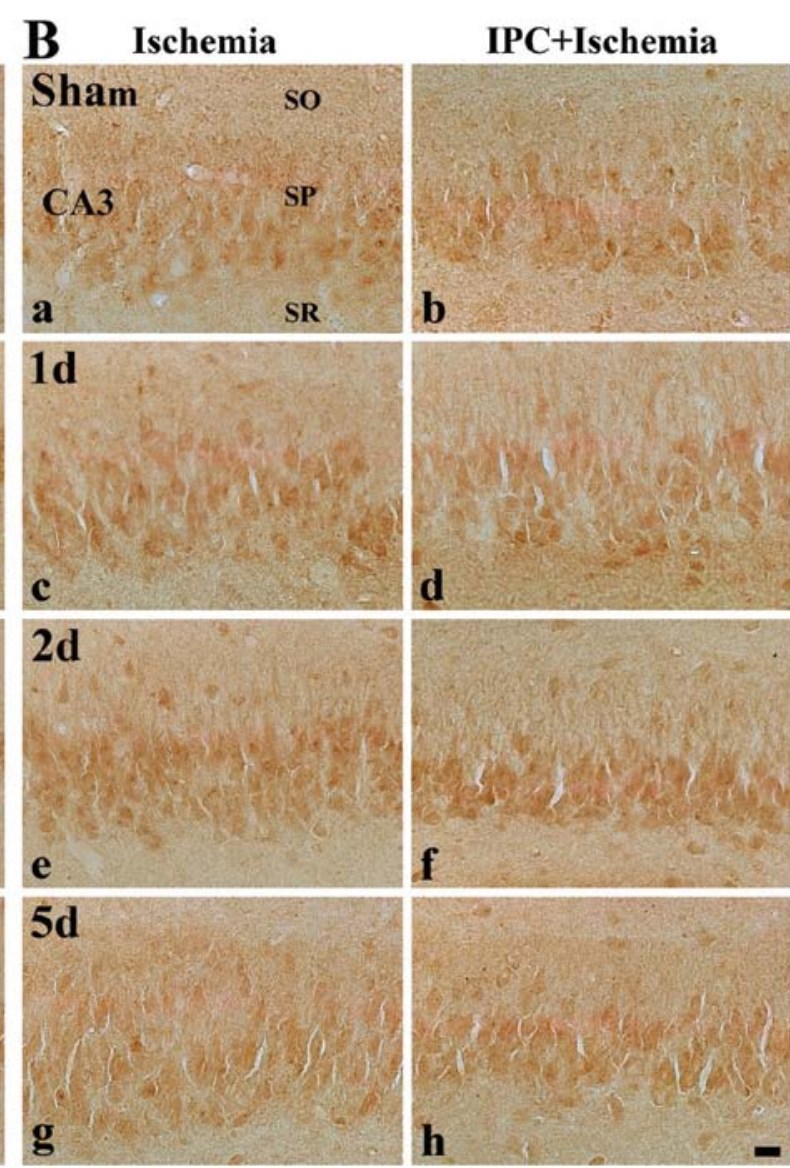
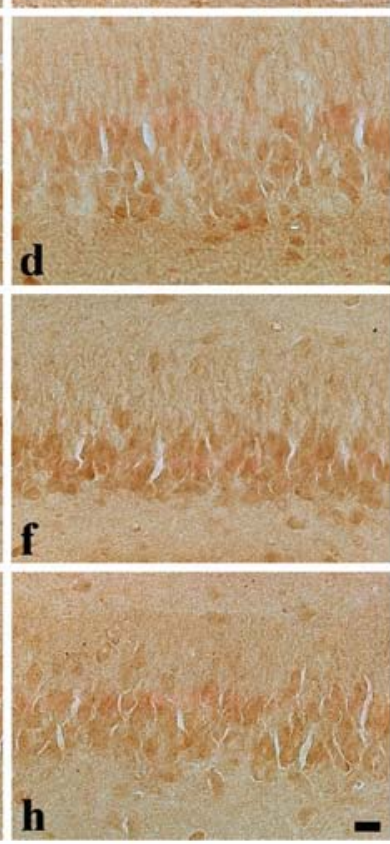

Figure 3. Immunohistochemistry for KYNA in (A) the CA1 region and (B) CA2/3 region of the ischemia-operated (first and third columns) and IPC + ischemiaoperated (second and fourth columns) groups at (a and b) sham, (c and d) 1 day ( $1 \mathrm{~d})$, (e and f) 2 days ( $2 \mathrm{~d}$ ) and ( $\mathrm{g}$ and h) 5 days $(5 \mathrm{~d})$ following ischemia-reperfusion. KYNA immunoreactivity was easily detected in the stratum pyramidale (SP) in the sham-operated group. In the CA1 region, KYNA immunoreactivity in the SP (asterisk) was increased at 1 day post-ischemia, decreased at 2 days post-ischemia and markedly decreased (reached its lowest level) at 5 days post-ischemia However, in the IPC + sham-operated group, KYNA immunoreactivity was similar to that in the sham-operated group, and the immunoreactivity was increased in the IPC + ischemia-operated group. KYNA immunoreactivity in the SP of the CA2/3 region was not significantly altered in all groups. KYNA, kynurenic acid; CA1, region I of hippocampus propern; IPC, ischemic preconditioning; SO, stratum oriens; SR, stratum radiatum. Scale bar, $50 \mu \mathrm{m}$.

IPC-mediated effect on KYNA levels. In the present study, we examined KYNA protein levels in the hippocampal CA1 homogenates at 2 and 5 days, when the immunohistochemical data were significantly altered following ischemia-reperfusion in both groups subjected to IPC. Western blot analysis revealed that the changes in the protein expression pattern of KYNA in the CA1 region following ischemia-reperfusion were similar to those observed in the immunohistochemical data (Fig. 4). 
Table II. Semi-quantification of the immunoreactivity of KYNA in pyramidal cells in the hippocampal CA1 and CA2/3 regions in the ischemia-operated and IPC + ischemia-operated groups.

Time after ischemia-reperfusion

\begin{tabular}{lccccccc}
\cline { 3 - 7 } Antibody & Region & Groups & Category & Sham & 1 day & 2 days & 5 days \\
\hline KYNA & CA1 & Ischemia & CSP & + & ++ & \pm & \pm \\
& & IPC + ischemia & CSP & + & ++ & ++ & ++ \\
& CA3 & Ischemia & CSP & + & + & + & + \\
& & IPC + ischemia & CSP & + & + & + & + \\
\hline
\end{tabular}

Immunoreactivity was scored as,,- \pm+ or ++ , representing no staining, weakly positive, moderate or strong, respectively. CSP, cells in the stratum pyramidale; IPC, ischemic preconditioning; KYNA, kynurenic acid.
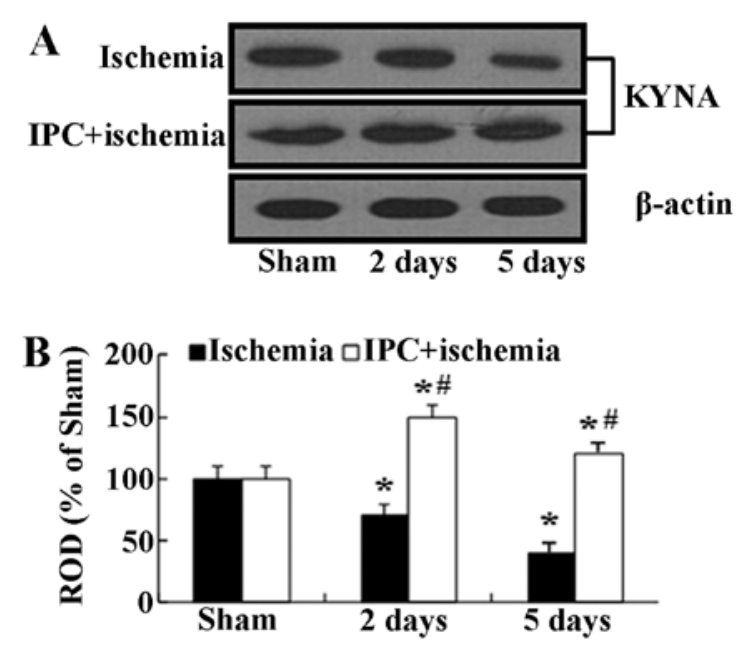

Figure 4. (A) Western blot analysis for kynurenic acid (KYNA) in the hippocampal CA1 region derived from the ischemia-operated and IPC + ischemia-operated groups. (B) Relative optical density (ROD) as percentage values of the western blot band is also represented ( $\mathrm{P}<0.05$ vs. sham-operated group; ${ }^{\#} \mathrm{P}<0.05$ vs. ischemia-operated group). The bars indicate the means \pm SEM.

In the ischemia-operated group, the KYNA protein levels were significantly decreased at 2 days and reached their lowest levels at 5 days following ischemia-reperfusion. On the other hand, in the IPC + sham-operated group, the KYNA protein levels were slightly increased compared with the sham-operated group, and the levels in the IPC + ischemia-operated-group were similar to those in the IPC + sham-operated group (Fig. 4).

\section{Discussion}

IPC is defined as a brief non-injurious episode of ischemia that is able to protect the brain from a subsequent longer ischemic insult $(11,12)$. Kitagawa et al (32) reported the description of IPC in the brain using a gerbil model of global ischemia. IPC shows a tolerance, which has been termed 'ischemic tolerance' and is activated at different time points following IPC; however, the molecular mechanisms underlying ischemic tolerance are not yet fully understood (33-35).

In the present study, we chose to induce a brief period ( $2 \mathrm{~min}$ ) of IPC to avoid histological tissue damage. This brief IPC stimulus did not induce neuronal damage, as assessed by $\mathrm{CV}$ and NeuN staining, and F-J B histofluorescence, which are sensitive markers for the detection of acute neuronal injury (11). We found that, at 5 days post-ischemia, the CA1 pyramidal neurons showed typically neuronal cell death, as shown by $\mathrm{CV}$ and NeuN staining, and F-J B histofluorescence. However, the viable CA1 pyramidal neurons were significantly protected from transient ischemic injury by IPC. However, although IPC often provides strong neuroprotection against ischemic brain injury, the exact mechanisms involved need to be investigated in order to develop therapeutic strategies for ischemic stroke.

KYNA, which is produced by astrocytes and neurons (18), is an endogenous metabolite of the kynurenine pathway for tryptophan degradation and is an antagonist of both NMDA and $\alpha 7$-nicotinic acetylcholine receptors (16-18). It is known that the level of endogenous KYNA is altered in several neurodegenerative disorders (36-38). However, data regarding the concentration of endogenous KYNA following an ischemic insult are limited. A few studies have demonstrated no change in the hippocampal content of KYNA or its decrease at 4 days after transient global ischemia $(22,39,40)$. In the present study, we found that KYNA immunoreactivity in pyramidal neurons was evidently altered changed following ischemia and was hardly detectable in the ischemia-operated group 5 days post-ischemia.

In the present study, we found that KYNA immunoreactivity in the CA1 pyramidal neurons of the animals in the IPC + ischemia-operated-group was evidently maintained or even increased following transient ischemia. To the best of our knowledge, no studies on the effects of IPC on KYN expression have been published to date. Nevertheless, many researchers have suggested that a sufficient elevation in the KYNA content in the brain leads to a definite neuroprotective effect (41-44), although the therapeutic potential of KYNA is limited, as KYNA is hardly able to cross the blood-brain barrier (26).

Pyramidal neurons in the hippocampal CA1 region are particularly vulnerable to excitotoxic processes following transient forebrain ischemia. Excitatory amino acids (EAAs) play an important role in the pathogenesis of cerebral ischemia (45). Under ischemic conditions, the release of excess EAAs contributes to the overactivation of ionotropic NMDA receptors and $\alpha$-amino-3-hydroxy-5-methyl-4-isoxazolepropionic acid (AMPA) receptors, which mediate the excessive entry of $\mathrm{Ca}^{2+}$, initiating glutamate-induced excitotoxicity, and finally, these excitotoxic processes eventually lead to neuronal death in the 
hippocampal CA1 region $(46,47)$. Therefore, it has been shown that the blockade of the receptors for EAA (the antagonism of NMDA receptors in particular) effectively reduces neuronal damage following ischemia $(48,49)$. Therefore, it is likely that IPC elevates the KYNA concentration sufficiently enough to affect the co-agonist site of the NMDA receptors.

In conclusion, the main findings of the present study demonstrated that in the levels of KYNA in the pyramidal neurons of the hippocampal CA1 region in animals subjected to IPC were maintained or even increased following ischemia-reperfusion and suggest that the increase in KYNA expression induced by IPC is associated with the endogenous protective response of the brain to ischemic injury.

\section{Acknowledgements}

The authors would like express their gratitude to Mr. Seung Uk Lee for providing technical assistance. The present study was supported by the Basic Science Research Program through the National Research Foundation of Korea (NRF) funded by the Ministry of Science, ICT and Future Planning (NRF-2014R1A2A2A01005307), and by a Priority Research Centers Program grant (NRF-2009-0093812) through the National Research Foundation of Korea funded by the Ministry of Science, ICT and Future Planning.

\section{References}

1. Kirino T: Delayed neuronal death in the gerbil hippocampus following ischemia. Brain Res 239: 57-69, 1982.

2. Lin CS, Polsky K, Nadler JV and Crain BJ: Selective neocortical and thalamic cell death in the gerbil after transient ischemia Neuroscience 35: 289-299, 1990.

3. Schmidt-Kastner R and Freund TF: Selective vulnerability of the hippocampus in brain ischemia. Neuroscience 40: 599-636, 1991.

4. Abe K, Aoki M, Kawagoe J, et al: Ischemic delayed neuronal death. A mitochondrial hypothesis. Stroke 26: 1478-1489, 1995.

5. Imon H, Mitani A, Andou Y, Arai T and Kataoka K: Delayed neuronal death is induced without postischemic hyperexcitability: continuous multiple-unit recording from ischemic CA1 neurons. J Cereb Blood Flow Metab 11: 819-823, 1991.

6. Shelat PB, Coulibaly AP, Wang Q, Sun AY, Sun GY and Simonyi A: Ischemia-induced increase in RGS7 mRNA expression in gerbil hippocampus. Neurosci Lett 403: 157-161, 2006.

7. Vollenweider F, Bendfeldt K, Maetzler W, Otten U and Nitsch C: GABA(B) receptor expression and cellular localization in gerbil hippocampus after transient global ischemia. Neurosci Lett 395 $118-123,2006$

8. Hou ST and MacManus JP: Molecular mechanisms of cerebral ischemia-induced neuronal death. Int Rev Cytol 221: 93-148, 2002.

9. Ientile R, Caccamo D, Marciano MC, et al: Transglutaminase activity and transglutaminase mRNA transcripts in gerbil brain ischemia. Neurosci Lett 363: 173-177, 2004.

10. Yu S and Cai J: Effects of aniracetam on extracellular levels of transmitter amino acids in the hippocampus of the conscious gerbils: an intracranial microdialysis study. Neurosci Lett 339 : 187-190, 2003.

11. Schmued LC and Hopkins KJ: Fluoro-Jade B: a high affinity fluorescent marker for the localization of neuronal degeneration. Brain Res 874: 123-130, 2000.

12. Lehotsky J, Burda J, Danielisova V, Gottlieb M, Kaplan P and Saniova B: Ischemic tolerance: the mechanisms of neuroprotective strategy. Anat Rec (Hoboken) 292: 2002-2012, 2009.

13. Gidday JM: Cerebral preconditioning and ischaemic tolerance. Nat Rev Neurosci 7: 437-448, 2006

14. Kardesoglu E, Isilak Z, Uz O and Yiginer O: Ischemic conditioning: a current concept in reducing reperfusion injury. Chin Med J (Engl) 124: 480, 2011.
15. Swartz KJ, During MJ, Freese A and Beal MF: Cerebral synthesis and release of kynurenic acid: an endogenous antagonist of excitatory amino acid receptors. J Neurosci 10: 2965-2973, 1990.

16. Kemp JA, Foster AC, Leeson PD, et al: 7-Chlorokynurenic acid is a selective antagonist at the glycine modulatory site of the N-methyl-D-aspartate receptor complex. Proc Natl Acad Sci USA 85: 6547-6550, 1988.

17. Kessler M, Terramani T, Lynch G and Baudry M: A glycine site associated with $\mathrm{N}$-methyl-D-aspartic acid receptors: characterization and identification of a new class of antagonists. J Neurochem 52: 1319-1328, 1989.

18. Hilmas C, Pereira EF, Alkondon M, Rassoulpour A, Schwarcz R and Albuquerque EX: The brain metabolite kynurenic acid inhibits alpha7 nicotinic receptor activity and increases non-alpha7 nicotinic receptor expression: physiopathological implications. J Neurosci 21: 7463-7473, 2001.

19. Moroni F, Cozzi A, Sili M and Mannaioni G: Kynurenic acid: a metabolite with multiple actions and multiple targets in brain and periphery. J Neural Transm 119: 133-139, 2012.

20. Sas K, Csete K, Vecsei L and Papp JG: Effect of systemic administration of L-kynurenine on corticocerebral blood flow under normal and ischemic conditions of the brain in conscious rabbits. J Cardiovasc Pharmacol 42: 403-409, 2003.

21. Lugo-Huitron R, Blanco-Ayala T, Ugalde-Muniz P, et al: On the antioxidant properties of kynurenic acid: free radical scavenging activity and inhibition of oxidative stress. Neurotoxicol Teratol 33: 538-547, 2011.

22. Salvati P, Ukmar G, Dho L, et al: Brain concentrations of kynurenic acid after a systemic neuroprotective dose in the gerbil model of global ischemia. Prog Neuropsychopharmacol Biol Psychiatry 23: 741-752, 1999.

23. Cozzi A, Carpenedo R and Moroni F: Kynurenine hydroxylase inhibitors reduce ischemic brain damage: studies with (m-nitrobenzoyl)-alanine (mNBA) and 3,4-dimethoxy-[-N4-(nitrophenyl)thiazol-2yl]-benzenesulfonamide (Ro 61-8048) in models of focal or global brain ischemia. J Cereb Blood Flow Metab 19: 771-777, 1999.

24. Abo M, Yamauchi H, Suzuki M, Sakuma M and Urashima M: Facilitated beam-walking recovery during acute phase by kynurenic acid treatment in a rat model of photochemically induced thrombosis causing focal cerebral ischemia. Neurosignals 15: 102-110, 2006.

25. Germano IM, Pitts LH, Meldrum BS, Bartkowski HM and Simon RP: Kynurenate inhibition of cell excitation decreases stroke size and deficits. Ann Neurol 22: 730-734, 1987.

26. Fukui S, Schwarcz R, Rapoport SI, Takada Y and Smith QR: Blood-brain barrier transport of kynurenines: implications for brain synthesis and metabolism. J Neurochem 56: 2007-2017, 1991.

27. Lee $\mathrm{CH}$, Park JH, Cho JH, et al: Changes and expressions of Redd1 in neurons and glial cells in the gerbil hippocampus proper following transient global cerebral ischemia. J Neurol Sci 344: 43-50, 2014.

28. Lee CH, Park JH, Choi JH, Yoo KY, Ryu PD and Won MH: Heat shock protein 90 and its cochaperone, p23, are markedly increased in the aged gerbil hippocampus. Exp Gerontol 46: 768-772, 2011.

29. Nakamura H, Katsumata T, Nishiyama Y, Otori T, Katsura K and Katayama Y: Effect of ischemic preconditioning on cerebral blood flow after subsequent lethal ischemia in gerbils. Life Sci 78: 1713-1719, 2006.

30. Lee $\mathrm{CH}$, Park JH, Yoo KY, et al: Pre- and post-treatments with escitalopram protect against experimental ischemic neuronal damage via regulation of BDNF expression and oxidative stress. Exp Neurol 229: 450-459, 2011.

31. Lee JC,Kim IH, ChoGS, etal: Ischemic preconditioning-induced neuroprotection against transient cerebral ischemic damage via attenuating ubiquitin aggregation. J Neurol Sci 336: 74-82, 2014.

32. Kitagawa $\mathrm{K}$, Matsumoto $\mathrm{M}$, Kuwabara $\mathrm{K}$, et al: 'Ischemic tolerance' phenomenon detected in various brain regions. Brain Res 561: 203-211, 1991.

33. Dhodda VK, Sailor KA, Bowen KK and Vemuganti R: Putative endogenous mediators of preconditioning-induced ischemic tolerance in rat brain identified by genomic and proteomic analysis. J Neurochem 89: 73-89, 2004.

34. Stenzel-Poore MP, Stevens SL, King JS and Simon RP: Preconditioning reprograms the response to ischemic injury and primes the emergence of unique endogenous neuroprotective phenotypes: a speculative synthesis. Stroke 38: 680-685, 2007. 
35. Feng Z, Davis DP, Sasik R, Patel HH, Drummond JC and Patel PM: Pathway and gene ontology based analysis of gene expression in a rat model of cerebral ischemic tolerance. Brain Res 1177: 103-123, 2007.

36. Kaminski RM, Zielinska E, Dekundy A, van Luijtelaar G and Turski W: Deficit of endogenous kynurenic acid in the frontal cortex of rats with a genetic form of absence epilepsy. Pol J Pharmacol 55: 741-746, 2003.

37. Stone TW: Kynurenines in the CNS: from endogenous obscurity to therapeutic importance. Prog Neurobiol 64: 185-218, 2001

38. Zadori D, Klivenyi P, Szalardy L, Fulop F, Toldi J and Vecsei L: Mitochondrial disturbances, excitotoxicity, neuroinflammation and kynurenines: novel therapeutic strategies for neurodegenerative disorders. J Neurol Sci 322: 187-191, 2012.

39. Saito K, Nowak TS Jr, Markey SP and Heyes MP: Mechanism of delayed increases in kynurenine pathway metabolism in damaged brain regions following transient cerebral ischemia. J Neurochem 60: 180-192, 1993.

40. Saito K, Nowak TS Jr, Suyama K, et al: Kynurenine pathway enzymes in brain: responses to ischemic brain injury versus systemic immune activation. J Neurochem 61: 2061-2070, 1993.

41. Stone TW: Development and therapeutic potential of kynurenic acid and kynurenine derivatives for neuroprotection. Trends Pharmacol Sci 21: 149-154, 2000.
42. Schwarcz R and Pellicciari R: Manipulation of brain kynurenines: glial targets, neuronal effects, and clinical opportunities. J Pharmacol Exp Ther 303: 1-10, 2002.

43. Vamos E, Pardutz A, Klivenyi P, Toldi J and Vecsei L: The role of kynurenines in disorders of the central nervous system: possibilities for neuroprotection. J Neurol Sci 283: 21-27, 2009.

44. Zadori D, Klivenyi P, Vamos E, Fulop F, Toldi J and Vecsei L: Kynurenines in chronic neurodegenerative disorders: future therapeutic strategies. J Neural Transm 116: 1403-1409, 2009.

45. Lipton SA and Rosenberg PA: Excitatory amino acids as a final common pathway for neurologic disorders. N Engl J Med 330: 613-622, 1994.

46. Dirnagl U, Iadecola C and Moskowitz MA: Pathobiology of ischaemic stroke: an integrated view. Trends Neurosci 22: 391-397, 1999.

47. Endres $M$ and Dirnagl U: Ischemia and stroke. Adv Exp Med Biol 513: 455-473, 2002.

48. Lai TW, Zhang S and Wang YT: Excitotoxicity and stroke: identifying novel targets for neuroprotection. Prog Neurobiol 115: 157-188, 2014.

49. Hoyte L, Barber PA, Buchan AM and Hill MD: The rise and fall of NMDA antagonists for ischemic stroke. Curr Mol Med 4: 131-136, 2004. 